\section{artigo original}

\author{
Marilza C.L. Ezabella \\ César $\Upsilon$. Hayashida \\ Neusa M.A. Abelin \\ Sérgio P.A. Toledo
}

\title{
Carcinoma Medular da Tireóide
}

$\mathrm{O}$ CARCINOMA MEdular DA TIREóIDE (CMT), identificado inicialmente como entidade clínico-patológica, em 1959, por Hazard e cols.(1) deriva das células $\mathrm{C}$, produtoras de calcitonina. Tem características específicas como presença de material amilóide, alta incidência de metástases em linfonodos cervicais e grau intermediário de malignidade, entre o carcinoma anaplásico e o carcinoma papilífero. Apesar de sua baixa incidência ( 3 a $12 \%$ dos carcinomas da tireóide), tem características bastante peculiares: 1) deriva das células $C$, originárias da crista neural, em contraste com as células foliculares; 2) produz uma grande variedade de substâncias bioativas, entre clas a calcitonina (CT), normalmente produzida pelas células $\mathrm{C} ; 3$ ) pode ocorrer de maneira esporádica (75 a 90\%), ou sob a forma familiar (10 a 25\%), com herança autossômica dominante. Nesta última, está geralmente associado a outras patologias, como parte das neoplasias endócrinas múlitiplas tipo 2A e 2B (NEM 2A e NEM 2B). Pode também se apresentar sob a forma familiar isolada (CMTF)(2-8) (Tabela 1 ).

Disciplina de Endocrinologia

Hospital das Clínicas da

Faculdade de Medicina da USP
Tabela 1. Tipos de carcinoma medular da tireóide.

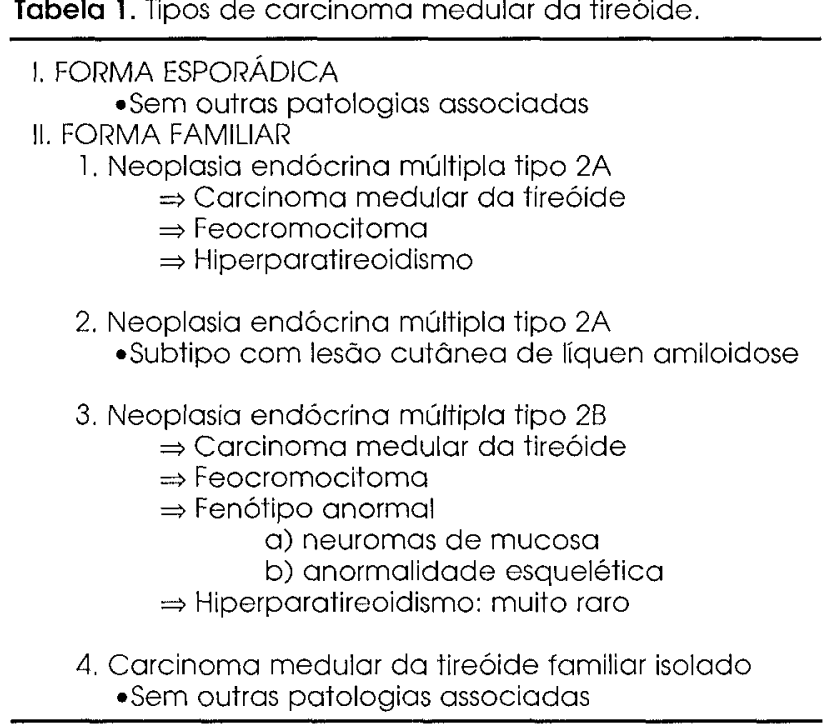

O CMT tem sido diagnosticado com freqüiência progressivamente maior, principalmente a sua forma hereditária, graças aos métodos de rastreamento entre familiares de indivíduos afetados. Assim, a forma hereditária chega a corresponder a até $25 \%$ dos casos em algumas séries(9). 


\section{ETIOLOGIA}

O CMT hereditário apresenta herança autossômica dominante, com alta penetrância e expressividade variável (10) Segundo Wolfe e cols. (11), a alteração patológica inicial do CMT hereditário é um aumento progressivo, multifocal, no número de células C localizadas nas porções médias dos lobos laterais da tireóide. Subseqüientemente, múltiplos focos microscópicos pré-invasivos de CMT se desenvolvem a partir destas áreas de hiperplasia de células C (HCC). O crescimento desses focos leva, então, ao aparecimento dos tumores visíveis macroscopicamente. Baseando-se na teoria de Knudson, dois eventos mutacionais na carcinogênese, Jackson e cols. $(12,13)$ propuseram que a HCC seria o resultado do primeiro evento genético mutacional herdado e uma mutação somática subseqüente levaria à transformação maligna destas células hiperplásicas. Baylin e cols. (14), empregando técnicas com glicose-6-fosfato desidrogenase, forneceram evidências de que a mutação inicial herdada, que ocorria na crista neural no início da vida fetal, produzia clones múltiplos de células suscetíveis à transformação neoplásica. Cada tumor formado posteriormente seria, portanto, resultado de uma ou mais alterações mutacionais envolvendo um clone de células suscetiveis (15). Em 1987, Simpson e cols. (16) e Mathew e cols. (17) localizaram o gene da NEM $2 \mathrm{~A}$ na região pericentromérica do cromossomo 10. Segundo Nelkin e cols.(18), esta alteração cromossômica poderia levar à hiperplasia policlonal das células C (e das células adrenais cromafins). Eventos genéticos posteriores, em que algumas células hiperplásicas progrediriam para formar carcinomas clonais, poderiam envolver locus cromossômicos diferentes dos que apresentam o defeito de linhagem germinativa no cromossomo 10 . Neste sentido, anomalias citogenéticas no cromossomo 1 têm sido descritas no CMT e no feocromocitoma. Em 1993, dois grupos de pesquisadores descreveram mutações germinativas (presentes em todas as células nucleadas) no proto-oncogene RET, associadas à NEM $2 \mathrm{~A}$ e ao CMTF $(19,20)$. O RET é parte da família do gene do receptor tirosina quinase que codifica uma molécula composta por um domínio extracelular (receptor), transmembrana e domínio intracelular (domínio catalítico, constituindo a tirosina quinase propriamente dita) (Figura 1.11). Desconhece-se a função deste proto-oncogene, assim como a substância ligante e o substrato endógeno da proteína codificada por este gene. Sabe-se, entretanto, que mutaçōes puntuais em um dos cinco resíduos cisteína nos códons $609,611,618,620$ e 634 estão associadas à NEM 2A e ao CMTF. Das 20 mutações conhecidas nesses resíduos, 15 resultam em alterações em sítios de reconhecimento de restrição dentro dos exons 10 ou 11 .

Quanto à NEM 2B, foi descrita uma mutação também germinativa, com troca de metionina por treonina no códon 918, localizado dentro do núcleo catalítico do domínio da tirosina quinase do RET $(21,22,23$ ) (Figura 1.11). Mutações no RET foram descritas apenas em um pequeno número de casos de CMT esporádico $(19,22,23)$

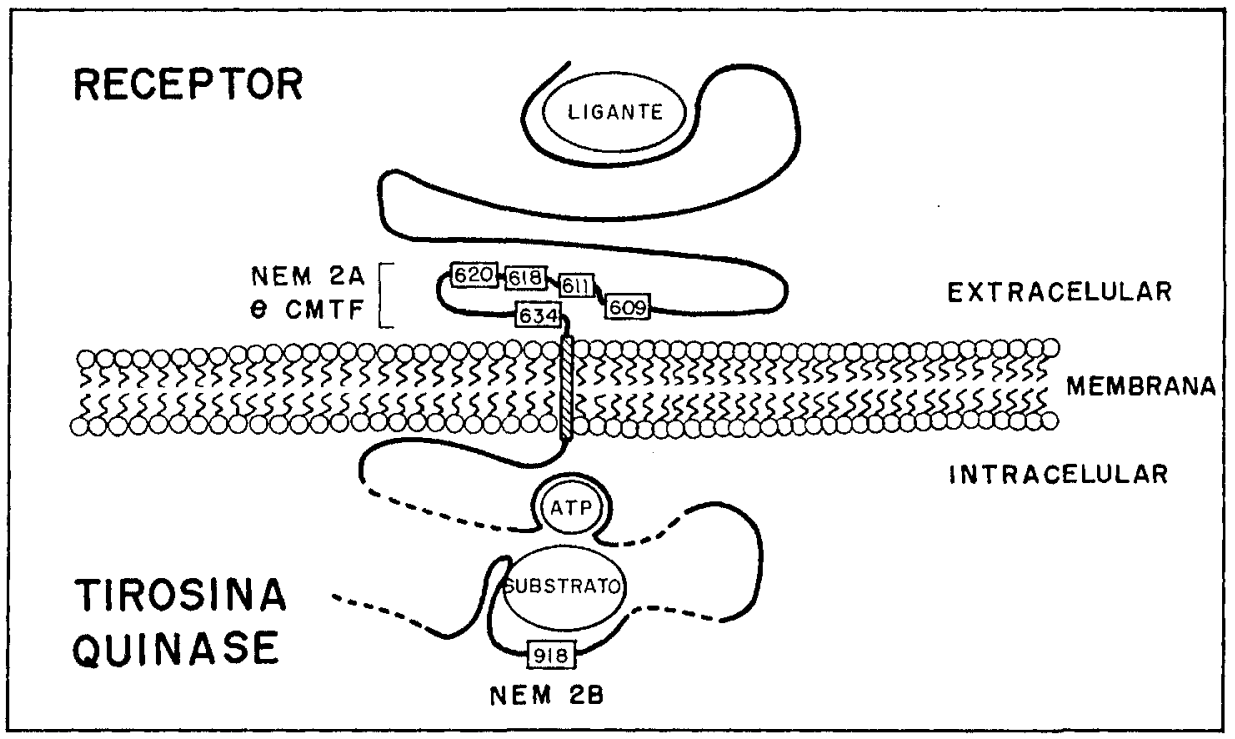

Figura 1. Representação da proteína RET. Mutações nos resíduos cisteina $609,611,618,620$ e 634 estão associadas a NEM 2A e CMTF. A mutação Met918 $\rightarrow$ Tre está associada a NEM 2B (Modificado de Wells S.A. Jr.(88)) 
$\mathrm{Na}$ NEM 2A, constatou-se que pacientes com troca de cisteína por arginina no códon 634 (TGC>CGC) apresentam maior propensão à doença da paratireóide do que aqueles com outras mutações neste mesmo códon (24). Assim, a variabilidade das manifestações clínicas observadas entre as diferentes famílias afetadas pode ser explicada pelos diferentes tipos de mutação que podem ocorrer em um mesmo gene. A deteç̧ão dessas mutações permite identificar indivíduos sob risco da doença (NEM 2A, NEM 2B e $\mathrm{CMTF}$ ) (ver rastreamento genético).

Rastreamento Familiar de CMT

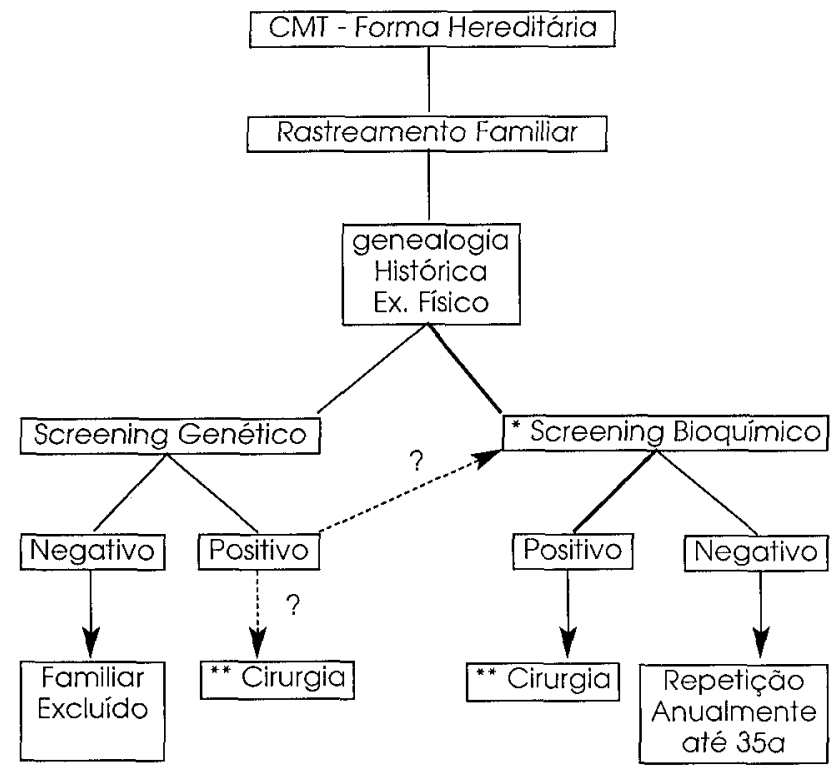

* Screening Bioquímico: testes de Estímulos para CT. Pesquisa de Feocromocitoma e Hiperparatireoidismo

* Cirurgia: Tireoidectomia Total + Limpeza Ganglionar Profilática + Localização das Paratireóides

\section{PATOBIOLOGIA}

Vários peptídeos e substâncias não peptídicas podem ser secretados pelo CMT, além de seu produto específico de secreção, a CT (25) (Tabela 2). Foram descritas secreção de ACTH (podendo causar síndrome de Cushing em 1 a $2 \%$ dos casos); precursor de ACTH, a pró-opiomelanocortina; beta-endorfina; beta-lipotrofina; MSH; hormônio liberador da corticotrofina; fator liberador de prolactina; somatostatina; substância $\mathrm{P}$; neurotensina; fator de crescimento do nervo; peptídeo liberador da gastrina; serotonina; melanina; histaminase; enolase neurônio específica e antígeno carcinoembriogênico. Este está presente também nas células $C$ hiperplásicas e normais.
TABELA 2 . Produtos secretados pelo CMT

1. Calcitonina

2. Outros hormônios polipeptídeos ACTH Endorfina Somatostatina VIP

3. Aminas bioativas e enzimas Dopamina Dopadescarboxilase Histaminase Serotonina

4. Outras substâncias Antigeno Carcinoembriogênico (CEA) Melanina

Fator de crescimento do nervo Prostaglandinas

\section{PATOLOGIA}

O CMT apresenta-se como uma massa sólida, brancoacinzentada, freqüentemente dura, bem circunscrita, porém não encapsulada. À microscopia óptica, apresenta células com citoplasma granular abundante que, à microscopia eletrônica, correspondem a pequenos grânulos de secreção elétron-densos limitados por membrana. As células podem ser fusiformes, poligonais ou ovais, com núcleo central. As figuras mitóticas são pouco freqüentes. A presença de amilóide é considerada uma característica distintiva do CMT. Difere de outros tipos de amilóide encontrados em outras doenças; é formado, possivelmente, a partir da CT ou de pró-CT (26). A presença deste, apesar de indicativa de CMT, não é invariável, podendo estar ausente em cerca de $10 \%$ dos casos (25). Além disso, o amilóide foi demonstrado em casos esporádicos de carcinoma de tireóide que não o CMT $(27,28)$. Portanto, a presença de amilóide não deve ser considerada como principal critério diagnóstico para o CMT (25). Além da ausência de amilóide, o diagnóstico pode ser dificultado pela presença de padrōes de crescimento atípicos, como papilar (29), folicular (30), tubular (31) e outros (32). Além disso, foram relatados vários casos de tumores exibindo tanto características medulares como foliculares $(33,34,35)$, assim como uma variante anaplásica do CMT $(36,37)$. Há descrição de casos de CMT com imunorreatividade à CT e à tireoglobulina nas mesmas células neoplásicas (38). Metástases do CMT ocorrem nos linfonodos cervicais e mediastinais por disseminação linfática, sendo que mais de $50 \%$ dos pacientes apresentam tais metástases por ocasião do diagnóstico. A disseminação hematogênica ocorre mais tardiamente para ossos, figado e pulmões. 


\section{DOENÇAS ASSOCIADAS}

\section{Feocromocitoma}

O feocromocitoma ocorre em 20 a $50 \%$ dos indivíduos com NEM 2A, sendo detectado em aproximadamente $40 \%$ daqueles que desenvolvem CMT nestas famílias $(7,9)$. O feocromocitoma, nesses casos, é bilateral em cerca $80 \%$ dos casos. Quando unilateral, a medular adrenal contralateral é freqüentemente hiperplásica e um segundo feocromocitoma pode aparecer anos após a remoção de um aparente tumor unilateral (39). Os tumores são usualmente benignos porém casos malignos, com invasão capsular e metástases, já tenham sido descritos $(40,41)$.

\section{Hiperparatireoidismo}

Entre os indivíduos com NEM 2A, 10 a $20 \%$ desenvolvem hiperplasia ou adenomas múltiplos das paratireóides (9). Embora hipercalcemia severa seja rara, esses pacientes muitas vezes apresentam cálculos renais $(42,43)$. O achado histopatológico mais freqüente é hiperplasia das paratireóides, descoberto geralmente durante a cirurgia do CMT, mesmo em pacientes sem hiperparatireoidismo clínico ou bioquímico $(42,43)$. Anomalias da paratireóide não fazem parte da NEM 2B, mas os pacientes com este diagnóstico podem apresentar paratireóides hiperplásicas descobertas durante a cirurgia $(44,45)$.

\section{APRESENTAÇĀO CLÍNICA}

O diagnóstico do CMT esporádico é feito com maior freqüência na $5^{\mathrm{a}}$ ou $6^{\mathrm{a}}$ década, com uma relação sexo feminino/masculino de, aproximadamente, 1,5:1. Apresenta-se, em geral, como um nódulo de tireóide único, indolor, firme e regular. Quando o nódulo é palpável, já se detecta metástases em linfonodos cervicais em cerca de metade dos casos.

O CMT hereditário, familiar, tem pico de incidência mais precoce, na $2^{a}$ ou $3^{\mathrm{a}}$ década, e pode ocorrer em crianças. Atinge igualmente ambos os sexos. Seu diagnóstico pode ser feito, precocemente, em uma fase pré-clínica, através de rastreamento genético ou bioquímico $(46,47)$

Os sinais e sintomas, tanto na forma esporádica como na hereditária, estão relacionados a: 1) extensão do crescimento tumoral local; 2) extensão e localização das metástases à distância e 3) efeitos dos produtos de secreção do tumor. Ao exame fisico, o CMT é indistingüível de nódulos de tireóide de outra natureza.

Sintomas como disfagia, rouquidão e pressão local são pouco freqüentes e dependem do tamanho e invasibilidade local do CMT. Metástase para linfonodos cervicais podem estar presentes precocemente, mesmo com lesões primárias pequenas, menores que $1,5 \mathrm{~cm}$ de diâmetro. Além das metástases cervicais, mais frequientes, outros locais, em ordem decrescente de freqüência, podem ser atingidos, como mediastino, pulmões, fígado, ossos, cérebro, adrenais, ovários e pleura $(7,8)$.

Substâncias bioativas produzidas pelo CMT (ver Tabela 2) podem ocasionalmente provocar algumas manifestações conhecidas como síndromes paraneoplásicas (48). Destas, a diarréia é a mais comum, ocorrendo em cerca de $30 \%$ dos casos, tanto na forma hereditária como na esporádica da neoplasia. A causa dessa manifestação é desconhecida; supõe-se que calcitonina, serotonina, prostaglandinas e VIP (vasoactive intestinal peptide) possam estar relacionados. Rubor facial foi relatado em $10 \%$ dos casos; prostaglandina e serotonina são os possíveis responsáveis pelo fenômeno. Há também relatos de síndrome de Cushing por produção de ACTH pelo tumor (49).

Manifestações específicas dos outros componentes da NEM 2A, como feocromocitoma e hiperparatireoidismo podem preceder, ocorrer simultaneamente, ou, mais comumente, ocorrer posteriormente ao CMT (50). Na NEM 2B, os neuromas de mucosa (congênitos ou de aparecimento na infầncia) e o hábito marfanóide são características fenotípicas que facilitam o diagnóstico. $O$ hiperparatireoidismo não ocorre na NEM 2B, ao contrário do feocromocitoma.

A Tabela 3 mostra as várias manifestações clínicas que podem ocorrer nas síndromes associadas ao CMT. O subtipo da NEM 2A com lesão cutânea é o mais raro $(51,52)$. Foram descritas cinco famílias em que havia associação de NEM $2 \mathrm{~A}$ e prurido hereditário localizado, que foi denominado líquen amiloidose cutânea. Esta lesão é caracterizada por prurido bilateral ou unilateral e lesões de pele liquenóides localizadas na região dorsal superior. Muitas vezes a lesão precede o diagnóstico de NEM 2A, servindo, portanto, como marcador fenotípico da síndrome (53).

\section{DIAGNÓSTICO}

Uma história familiar sugestiva, assim como a presença de feocromocitoma, hiperparatireoidismo, fenótipo anormal ou síndromes paraneoplásicas em um paciente com nódulo de tireóide, deve suscitar o diagnóstico de CMT hereditário. Caso contrário, os sinais e sintomas costumam ser inespecíficos, entrando no diagnóstico diferencial de nódulo de tireóide.

A cintilografia da tireóide com ${ }^{131}$ I ou com 
$99 \mathrm{mTc}$ mostra áreas tumorais com imagens negativas (nódulo frio). A cintilografia com metaiodobenzilguanidina ( $\left.{ }^{131} \mathrm{I}-\mathrm{MIBG}\right)$, ácido dimetil-succínico pentavalente ( ${ }^{99} \mathrm{Tc}$-DMSA-V), tálio $\left({ }^{201} \mathrm{Tl}\right)$, In-anti CEA e "Octreotide" pode apresentar imagem tumoral positiva. A disponibilidade destas substâncias mapeadoras é limitada; são empregadas na localização de metástases do CMT (54-58). A ultrassonografia cervical pode ser útil na caracterização de tumor multifocal e no envolvimento de linfonodos regionais.

O diagnóstico de CMT pode ser feito, em geral, pela punção aspirativa por agulha fina (PAAF) do nódulo tireoideano e posterior estudo imunocitoquímico para CT, além da detecção de níveis séricos elevados deste hormônio (59-61). A dosagem rotineira de CT sérica basal em pacientes com nódulos tireoideanos usualmente não é feita por ser economicamente inviável. Em nossa experiência, a PAAF sem a imunocitoquímica específica do material obtido tem sido de pouca utilidade, com erro diagnóstico freqüente (48). Apesar da falta de especificidade, o antígeno cárcino-embriônico (CEA) costuma também estar elevado e seus níveis séricos parecem estar dirctamente relacionados com a extensão do carcinoma (62-64). de hiperplasia das células $\mathrm{C}$, achados que distinguem a forma hereditária da esporádica deste carcinoma $(66,67)$ (Tabela 3).

\section{CONSIDERAÇÕES DIAGNÓSTICAS}

A distinção entre as formas esporádica e hereditária do CMT apresenta consequiências, não apenas para o paciente, mas também para os familiares. Sendo o padrăo de herança autossômico dominante, metade dos familiares de um caso de CMT hereditário apresenta risco de manifestar este carcinoma. Na NEM 2A existe ainda o risco relativo de feocromocitoma e de hiperparatireoidismo, que podem se manifestar anos após o CMT. O tratamento precoce do CMT, em fase pré-carcinoma (apenas hiperplasia de células C), ou em fases iniciais, leva habitualmente à cura.

O diagnóstico do CMT hereditário pode apresentar dificuldades. Uma história familiar positiva para CMT ou NEM é altamente indicativa, mas uma história negativa não afasta a possibilidade de carcinoma hereditário. Um paciente pode também representar a primeira mutação de CMT familiar (68) (Esquema para Diagnóstico de CMT).

Tabela 3. Apresentação clínica do carcinoma medular da tireóide.

\begin{tabular}{|c|c|c|c|c|c|c|}
\hline Tipos & $\begin{array}{l}\text { Familiares } \\
\text { Afetados }\end{array}$ & $\begin{array}{l}\text { Idade ao } \\
\text { diagnóstico }\end{array}$ & $\begin{array}{l}\text { Lesão } \\
\text { tireóidea }\end{array}$ & $\begin{array}{l}\text { Hiperplasia } \\
\text { de Células C }\end{array}$ & $\begin{array}{l}\text { Outras patologias } \\
\text { associadas }\end{array}$ & Agressividade \\
\hline Esporádico & - & $5^{\circ}-6^{\mathrm{o}}$ década & Unilateral & - & Nenhuma & Variável \\
\hline NEM $2 A$ & + & $2^{a}-3^{a}$ década & Bilateral & + & $50 \%$ feocromocitoma & Variável \\
\hline $\begin{array}{l}\text { NEM 2A com } \\
\text { amiloidose } \\
\text { liquen } \\
\text { cutânea }\end{array}$ & + & $1^{\mathrm{a}}-2^{\mathrm{q}}$ década & Bilateral & + & $\begin{array}{l}40 \% \text { hiperparatireoidismo } \\
50 \% \text { feocromocitoma } \\
40 \% \text { hiperparatireoidismo } \\
100 \% \text { lesão cutânea } \\
50 \% \text { feocromocitoma }\end{array}$ & Variável \\
\hline NEM 2B & + & $l^{\mathrm{g}}-2^{\mathrm{a}}$ década & Bilateral & + & $100 \%$ neuromas de mucosa & + agressiva \\
\hline CMTF isolado & + & $4^{a}-5^{\circ}$ década & Bilateral & + & $\begin{array}{l}100 \% \text { hábito marfanóide } \\
\text { raro hiperparatireoidismo } \\
\text { nenhuma }\end{array}$ & - agressiva \\
\hline
\end{tabular}

O diagnóstico pré-operatório do CMT é importante, possibilitando uma abordagem cirúrgica própria, com ressecção rotineira dos linfonodos cervicais centrais. Permite também detectar doenças associadas, particularmente o feocromocitoma, que deve ser sempre tratado antes do CMT, pelos seus riscos inerentes (65).

O exame anátomo-patológico da tireóide deve ser minucioso, com cortes histológicos não apenas do tumor, mas também de toda a glândula, e acompanhado, rotineiramente, de imunohistoquímica para calcitonina. Além da confirmação diagnóstica, pode-se assim determinar a clonalidade do CMT (policlonal e, portanto, multifocal na forma hereditária) e a presença

\section{rastreamento familiar}

Os dados acima expostos indicam que o rastreamento familiar deve ser feito ou tentado em todos os casos de CMT (69-71). A história familiar, apesar de pouco sensível, deve ser detalhada e voltada, particularmente, para manifestações relacionadas ao CMT, ao feocromocitoma e ao hiperparatireoidismo. Assim, uma detalhada genealogia deve ser obtida, incluindo dados como presença de nódulo cervical, hipertensão arterial, nefrolitíase e doença cardiovascular.

Os familiares devem ser submetidos a um protocolo clínico e, quando disponível, a teste genético específico para deteç̧ão de NEM 2A, NEM 2B, ou CMTF. Na impossibilidade deste, devem ser submeti- 


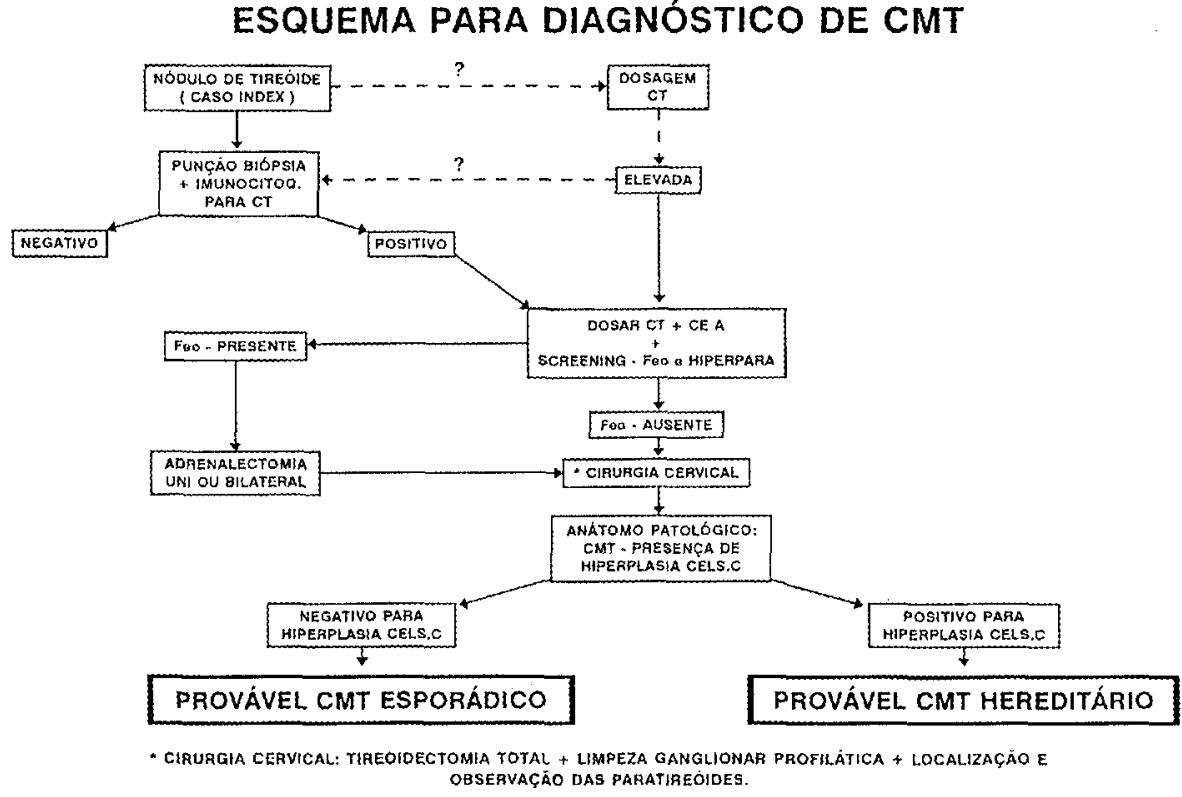

dos a testes bioquímicos provocativos da calcitonina, uma vez que a simples determinação da calcitonina sérica basal é pouco sensível nas fases de hiperplasia das células $\mathrm{C}$ ou mesmo no CMT inicial (72). Conforme já salientado, a tireoidectomia total realizada nestas fases apresenta os maiores índices de cura, próximos de $100 \%$.

\section{Rastreamento bioquímico}

O CMT, sendo derivado das células $\mathrm{C}$, tem na CT o seu marcador tumoral específico. A determinação de seus níveis séricos tem, portanto, importância fundamental no seu diagnóstico e seguimento. Além disso, este procedimento tem sido a base para o rastreamento familiar bioquímico da doença. A CT sérica costuma estar elevada nos casos de CMT que se apresentam como nódulo de tireóide palpável. A CT pode entretanto estar normal na fase pré-clínica, de hiperplasia de células C, acompanhada ou não de focos microscópicos do carcinoma. Testes farmacológicos provocativos de CT têm sido empregados justamente para identificar indivíduos nesta fase (72-74).

$\mathrm{O}$ teste de estímulo com pentagastrina, um análoģo sintético da gastrina, com cálcio ou com a combinação dos dois são os empregados mais freqüentemente. No primeiro teste, é feita administração endovenosa de $0,5 \mathrm{mg} / \mathrm{kg}$ de pentanúcleo gastrina, em 5 segundos. Amostras de sangue são coletadas antes do teste (basal) e após 2, 5 e 10 minutos. Apresenta como efeitos colaterais mais freqüentes sensação de aperto retro-esternal e epigastralgia, ambos bastante fugazes. É contra-indicado em portadores de doença cárdio-vascular, gestantes, idosos e crianças menores de 4 anos de idade (75).

A infusão de cálcio se faz por via endovenosa, durante 1 minuto, na dose de $2 \mathrm{mg}$ de cálcio $/ \mathrm{kg}$. Os tempos de coleta de sangue são os mesmos do teste da pentagastrina. O teste pode provocar ondas de calor e, ocasionalmente, náuseas. Deve ser evitado em indivíduos já hipercalcêmicos, e com hiperparatireoidismo (76).

O teste combinado de cálcio e pentagastrina é o mais sensível, com baixo índice de respostas falso-negativas. Administra-se cálcio e, imediatamente depois, pentagastrina, em dose, via e coleta de sangue semelhantes às descritas acima para cada substância. A amplificação da resposta da CT assim obtida parece não causar aumento dos efeitos colaterais.

Um teste positivo deve sempre ser confirmado por um segundo antes de se indicar tireoidectomia. Parentes de primeiro grau de um caso índice de CMT são submetidos a um destes testes a partir de 3 a 5 anos de idade, e repete-se anualmente até os 35 anos, ou até a positividade do mesmo $(77,78)$.

Diferentes ensaios trazem diferentes limites de normalidade da CT sérica. Assim, a interpretação dos resultados dos testes deve ser padronizada para cada laboratório. Crianças normalmente apresentam níveis mais elevados, assim como adultos do sexo masculino, tabagistas, portadores de pneumopatias crônicas, de insuficiência renal, de pancreatite, de ileíte regional, estados de hipergastrinemia e estados hipercalcêmicos.

Na Unidade de Endocrinologia Genética da FMUSP conseguimos estabelecer os limites de nor- 
malidade para os valores séricos de CT, basais e sob estímulo $(79,80)$, utilizando técnica de radioimunoensaio policlonal, cuja sensibilidade de detecção foi de $13,96 \pm 5,91 \mathrm{pg} / \mathrm{ml}$, com baixos coeficientes de variação, tanto intra como interensaio $(8,7 \%$ e $12,1 \%)$, respectivamente.

Os valores basais de calcitonina de 146 voluntários, distribuídos em diversos grupos de análise, com idades variando de 12 a 85 anos, foram, em média, de $22,88 \pm 12,21 \mathrm{pg} / \mathrm{ml}$. Diferenças significantes $(p<0,01)$ foram encontradas somente no grupo de gestantes em seu $3^{\mathbf{0}}$ trimestre de gestação $(29,28 \pm$ $17,02 \mathrm{pg} / \mathrm{ml})$, e entre os sexos $(\mathrm{p}<0,05)$, nos adultos pertencentes ao grupo etário acima de 50 anos (homens: $35,03 \pm 16,12 \mathrm{pg} / \mathrm{ml}$ contra mulheres: $22,78 \pm$ $15,48 \mathrm{pg} / \mathrm{ml})$.

A resposta ao teste de estímulo combinado (cálcio + pentagastrina) realizado em 52 indivíduos normais ( 18 homens e 34 mulheres), não revelou valores de estímulo acima de $251 \mathrm{pg} / \mathrm{ml}$, sendo que as mulheres revelaram respostas mais baixas (pico de resposta $156 \mathrm{pg} / \mathrm{ml}$ ) e $17 \%$ delas não apresentaram nenhuma resposta ao estímulo.

Desta maneira, respostas de calcitonina ao teste combinado, acima de $251 \mathrm{pg} / \mathrm{ml} \mathrm{em}$ pacientes de risco (familiares de CMT), utilizando este tipo de ensaio e seguindo os critérios de controle de qualidade estabelecidos em nosso laboratório, são consideradas fortemente suspeitas de portador de CMT (81-84).

\section{Rastreamento genético}

A identificação das mutações específicas no RET associadas com NEM 2A, NEM 2B ou CMTF propiciou um método moderno e de grande importância para se determinar quais membros de uma família herdaram o alelo mutante e estão, portanto, sob risco da doença $(85,86)$. Dois métodos diretos de teste de DNA para detecção destas mutações são atualmente empregados. Ambos dependem da análise do DNA extraído de leucócitos. Se a mutação associada a NEM $2 \mathrm{~A}$ cria ou abole um sítio de restrição de reconhecimento de uma endonuclease, a digestão do DNA é um meio relativamente fácil de identificar os familiares afetados. Em casos de mutações que não alteram sítios de restrição, o seqüenciamento direto, tecnicamente mais trabalhoso, é necessário para identificação do indivíduo sob risco.

Várias são as vantagens do teste direto do DNA em relação ao rastreamento bioquímico por testes provocativos de CT. O teste direto é feito apenas uma vez na vida de um paciente. Assim, familiares sob risco, mas que apresentam teste negativo para a mutação, não requerem testes genéticos ou bioquímicos adicionais, assim como seus descendentes. Aqueles que herdaram o alelo mutante necessitarão de aconselhamento genético para conhecerem o risco de desenvolver CMT e outras doenças associadas. Também devem ser explicados os riscos para seus filhos. Devese considerar tireoidectomia total nestes pacientes. Em contraste com os testes provocativos da CT, o teste direto do DNA não está associado a qualquer efeito colateral.

Alguns autores recomendam tireoidectomia total e ressecção dos linfonodos do compartimento central do pescoço baseadas apenas no teste do DNA (86). Outros recomendam testes provocativos da CT periódicos nestes portadores da mutação e tireoidectomia apenas naqueles em que o teste provocativo se mostrar alterado (87).

Não tem sido possível identificar mutações no RET em algumas famílias com NEM 2A ou CMTF (88). Até que as mutações nestas famílias sejam identificadas, é necessário estabelecer o diagnóstico pré-sintomático da herança da doença através de testes provocativos da CT ou, então, através de análise de haplotipos.

Em um paciente com CMT e sem história familiar de neoplasia endócrina, deve-se examinar cuidadosamente a tireóide ressecada, na tentativa de se caracterizar a presença de CMT bilateral, hiperplasia de células $\mathrm{C}$, ou ambos, já que estes achados histológicos são característicos da forma hereditária da neoplasia. (67). Se o paciente não apresentar qualquer evidência histórica, clínica ou histológica de CMT hereditário, a avaliação pós-operatória deve consistir de teste provocativo anual. Se houver evidência de CMT hereditário, como tumor bilateral, hiperparatireoidismo ou feocromocitoma, o paciente deve realizar o mesmo teste direto de DNA para se identificar a mutação específica no RET.

\section{TRATAMENTO}

A cirurgia é o tratamento de escolha no CMT. A tireoidectomia total deve ser realizada não apenas nos casos de CMT familiar, de origem policlonal e, portanto, multifocal, mas também no CMT aparentemente esporádico, devido à incerteza relacionada com este dado por ocasião da cirurgia. A tireoidectomia total deve ser sempre acompanhada de ressecção dos linfonodos cervicais da região central, delimitada pelo osso hióide, superiormente, pelos vasos inominados, inferiormente, e pelas jugulares internas, lateralmente, por ser local freqüente de metástases, muitas vezes não detectáveis 
macroscopicamente (89). Esta ressecção "profilática" dos linfonodos centrais se justifica, uma vez que metástases são aí encontradas em 50\% dos casos. Deve-se estudar amostras de linfonodos medianos das jugulares e, se estiverem comprometidos, deve-se realizar esvaziamento extenso e meticuloso do lado afetado (90-92).

As paratireóides devem ser reconhecidas durante a cirurgia. Aquelas macroscopicamente alteradas devem ser removidas. A retirada das paratireóides, com autotransplante parcial, está indicada naqueles casos com hiperparatireoidismo e aumento de todas as paratireóides $(93,94)$. Wells $(88)$ recomenda paratireoidectomia de rotina (com implante parcial em antebraço no NEM $2 \mathrm{~A}$ e no músculo esternocleidomastóide em CMT esporádico), independente do aspecto destas glândulas. Justifica este procedimento pelo comprometimento do suprimento sangüineo às paratireóides após uma cirurgia de tal amplitude e, no caso de NEM 2A, pela ocorrência futura de hiperparatireoidismo em 30 a $40 \%$ dos casos.

Outros métodos terapêuticos têm sido pouco eficazes. A radioterapia pode ser indicada nos casos inoperáveis, podendo levar à redução da massa tumoral e ao alívio dos sintomas obstrutivos. Entretanto, complicações como traqueíte e fibrose cervical limitam seu uso $(95,96)$.

A quimioterapia também não é habitualmente eficaz. Obteve-se alguma regressão tumoral com adriamicina (97). Outros quimioterápicos, quando usados em associação, como dacarbazina e 5-fluouracil podem retardar o avanço da doença. A combinação de ciclofosfamida, vincristina e dacarbazina parece ter reduzido o tamanho do tumor em um número bastante limitado de pacientes estudados (98).

O uso de um análogo sintético da somatostatina ("Sandostatin") resultou em melhora do estado geral e da diarréia. Entretanto, não interferiu na progressão do tumor ou das metástases $(99,100)$.

O uso terapêutico de radiofármacos ativamente captados pelo CMT, como meta-iodo-benzil-guanidina ( $\left.{ }^{99} \mathrm{I}-\mathrm{MIGB}\right)$ e ácido dimetil-succínico $\left({ }^{99} \mathrm{Tc}-\mathrm{V}\right.$ DMSA) encontra-se em fase experimental $(101,102)$.

O CMT é um bom modelo para estudo de células neoplásicas em cultura de tecidos (103,104). A avaliação dos níveis da CT em cultura, além de confirmar a etiologia do tumor, fornece informações sobre a ação de drogas nesta cultura, que pode ser de utilidade terapêtutica.

\section{SEGUIMENTO}

O critério mais aceito de cura do CMT é a normalização dos níveis séricos de CT, tanto basal como após estímulo. Pacientes com metástases em linfonodos cervicais freqüentemente não conseguem apresentar níveis normais de CT após cirurgia $(105,106)$. Neste aspecto, a presença de mais de três linfonodos comprometidos parece constituir fator significativo para a recorrência da doença (106). Estes dados mostram a importância do diagnóstico e tratamento precoces, como já salientado.

As metástases ocorrem habitualmente por via linfática, particularmente nos linfonodos cervicais, e no mediastino. $\mathrm{O}$ acometimento hematogênico, hepático, pulmonar, ósseo e de outros órgãos costuma ser tardio e variável.

A pesquisa dessas metástases pode ser feita pelos métodos usuais, como ultrassonografia, tomografia computadorizada e ressonância magnética das regiões que possam estar comprometidas, assim como cintilografia óssea. Radiofármacos especificamente captados pelo CMT, como ${ }^{131} \mathrm{I}-\mathrm{MIBG},{ }^{201} \mathrm{Tl}$ e ${ }^{99} \mathrm{TC}-\mathrm{V}-\mathrm{DMSA}$ também são utilizados e apresentam resultados variáveis (107-109). Apesar da disponibilidade de todo este aparato propedêutico, pequenos focos de CMT não são, freqüentemente, localizáveis. Nesta situação, o cateterismo venoso seletivo, com determinação de gradiente de concentração de CT no local suspeito em relação à periferia, pode ser útil (110-112).

Um paciente é considerado portador de doença metastática oculta quando apresenta CT sérica elevada c sem foco tumoral localizável. Esta situação não é rara no CMT e estes pacientes podem assim permanecer por período de tempo variável, às vezes bastante prolongado (113).

Há controvérsia em relação à conduta nesta situação. Alguns advogam uma reoperação da região cervical e do mediastino superior, em que se resseca, minuciosamente, os possíveis focos da doença oculta (114117). São necessários um tempo de seguimento e um número maior de casos até que se estabeleça a validade deste tipo de conduta (118). Outros defendem uma conduta expectante, indicando a reoperação apenas quando a doença for demonstrada clínica ou radiograficamente $(106,113)$. Essa conduta é justificada pela evolução indolente do CMT, com alguns pacientes clinicamente estáveis apesar de CT sérica elevada.

\section{PROGNÓSTICO}

O CMT hereditário parece apresentar prognóstico um pouco mais favorável do que o esporádico, exceto aquele associado a NEM 2B, de comportamento altamente agressivo. Este necessita, portanto, de intervenção cirúrgica o mais precoce possível. Neste senti- 
do, o fenótipo característico da NEM 2B deve servir de valioso auxiliar diagnóstico, já no período neonatal. O CMTF, por sua vez, costuma ser o menos agressivo, com um curso indolente.

Em uma grande série, de Saad e cols. (119), a sobrevida em 5 e 10 anos foi de $65 \%$ e $40 \%$ respectivamente, no CMT como um todo. Nesta série, apenas $3 \%$ dos pacientes com a forma hereditária do carcinoma morreram da doença, contra $40 \%$ na forma csporádica. É provável que esta grande diferença se deva ao diagnóstico precoce das formas hereditárias, graças ao rastreamento familiar.

Em uma outra séric com 249 pacientes, de Bergholm e cols. (120), a sobrevida, no mesmo período, foi de $79,9 \%$ e $68,6 \%$.

Os fatores associados a um pior prognóstico são: 1) idade acima de 40 anos; 2) metástases mediastinais; 3) cirurgia incompleta; 4) invasão capsular; 5) doença extra-nodal; 6) CEA sérico elevado e 7) imunohistoquímica heterogênea para CT, com correlação inversa entre positividade para CT e prognóstico (64,119-121). Pacini e cols. (122) sugeriram que a presença de somatostatina no CMT pode estar associada à sobrevida mais longa.

A correta identificação dos fatores prognósticos, com a determinação do grau de agressividade do CMT em cada caso, pode ser útil na seleção daqueles pacientes que irão se beneficiar com uma reintervenção cirúrgica agressiva.

\section{REFERÊNCIAS}

1. Hazard JB, Hawk WA, Crile Jr G Medullary (Solid) carcinoma of the thyroid - A clinicopathologic entily. J Clin Endocrinol 1959; 19:152-61.

2. Sipple JH. The association of pheochromocytoma with carcinoma of the thyroid gland. Am J Med 1961; 31:16365.

3. Schimke RN \& Hartmann WH Familial amyloid-producing medullary thyroid carcinoma and pheochromocytoma. Ann Intern Med 1965; 63:1027-39.

4. Sarosi $G$ \& Doe RP Familial occurrence of parathyroid adenomas, pheochromocytoma, and medullary carcinoma of the thyroid with amyloid stroma (Sipple's syndrome), Ann Int Med 1968; 68:1305-09.

5. Keiser HR, Beaven MA, Doppman J, Wells Jr S, Buja ML. Sipple's syndrome: medullary thyroid carcinoma. pheochromocytoma, and parathyroid disease Studies in a large family. Ann Intern Med 1973; 78:561-79.

6. Block MA, Roberts JP, Kadair RG, Seyfer AE, Hull SF, Nofeldt FD Multiple endocrine adenomatosis type lib Diagnosis and treatment. JAMA 1975; 234:710-14

7. Emmertsen K. Medullary thyroid carcinoma and calcitonin. Dan Med Bull 1985; 32: 1-28.
8. Gagel RF, Robinson MF, Donovan DT, Alford BR Clinical Review 44: Medullary thyroid carcinoma - recent progress. J Clin Endocrinol Metab 1993; 76:809-14.

9. Raue F, Rave KF, Graver A Multiple Endocrine Neoplasia type 2: Clinical features and screening. Endocrinol Metab Clin North Am 1994; 23:137-56.

10. Schimke RN Genetic aspects of multiple endocrine neoplasia. Ann Rev Med 1984, 35:25-31

11. Wolfe HJ, Melvin KEW, Cervi-Skinner SJ, Al Saadi AA, Juliar JF, Jackson CE, Tashijan AHJr C-cell hyperplasia preceding medullary thyroid carcinoma. N Engl J Med 1973; 289:437-41.

12. Knudson AGJr, Stron LC, Anderson DF Heredity and cancer in man. Prog Med Genet 1973; 9:133-58.

13. Jackson CE, Block MA; Greenawald KA, Tashjian AHJr The two-mutational-event theory in medullary thyroid carcinoma. Am J Hum Genet 1979; 31:704-10.

14. Baylin SB, Gann DS, Hsu SH Clonal origin of inherited medullary thyroid carcinoma and pheochromocytoma. Science 1976; 193:321-23.

15. Baylin SB, Hsu SH, Gann DS, Smallidge RC, Wells Jr SA Inherited medullary thyroid carcinoma: a final monoclonal mutation imposed on one of multiple clones of susceptible celis. Science 1978; 199:429-31.

16. Simpson NE, Kidd KK, Goodfellow PJ, McDermid H, Myers S, Kidd JR, Assignment of multiple endocrine neoplasia type $2 \mathrm{~A}$ to chromosome 10 by linkage. Nature 1987; 328:528-30

17. Mathew CGP, Chin KS, Easton DF, Thorpe K, Carter C, Liou $\mathrm{Gl}$, A linked genetic marker for multiple endocrine neoplasia type $2 \mathrm{~A}$ on chromosome 10. Nature 1987; 328:527-28.

18. Nelkin BD. Bustros AC, Mabry M, Baylin SB. The molecular biology of medullary thyroid carcinoma. JAMA 1989; 261:3730-135.

19. Donis-Keller H, Dou S, Chi D, Carlson KM, Toshima K, Lairmore. Mutations in the RET proto-oncogene are associated with MEN 2A and FMTC. Hum Molec Genet 1993; 2:851-56.

20. Mulligan LM, Kwok JBJ, Healey CS, Elsdon MJ, Eng C, Gardner E. Germ-line mutations of the RET proto-oncogene in multiple endocrine neoplasia type 2A. Nature 1993; 363:458-60.

21. Carlson KM, Dou S, Chi D. Scavarda N. Toshima K, Jackson CE. Single missence domain of the RET proto oncogene is associated with multiple endocrine neoplasia type 2B. Proc Natl Acad Sci USA 1994; 91:1579-83.

22. Hofstra RMW, Landsvater RM, Ceccherini I, Stulp RP Stelwagen T, Luo Y, A. mutation in the RET proto-oncogene associated with multiple endocrine neoplasia type $2 \mathrm{~B}$ and sporadic medullary thyroid carcinoma. Nature 1994; 367:375-76.

23. Eng C, Smith DP, Mulligan LM, Nagai MA, Healey CS, Ponder MA, Point mutation within the tyrosine kinase domain of the Ret proto-oncogene in multiple endocrine neoplasia type $2 \mathrm{~B}$ and related sporadic tumours. Hum Molec Genet 1994; 3:237-41.

24. Mulligan LM, Eng C, Healey CS, Clayton D. Kwok JBJ, Gardner E, et al. Specific mutations of the RET proto- 
oncogene are related to disease phenotype in MEN 2A anol FMTC. Nature Genet 1994; 6:70-4.

25. Saad MF, Ordonez NG, Rashid RK, Guido JJ, Hill CS. Hickey RC, et al. Medullary carcinoma of the thyroid. A study of the clinical features and prognostic factors in 161 patients. Medicine 1994; 63:319-342.

26. Sletten, K: Westermark, P. Natvig, JB Characterization of amyloid fibril proteins from medullary carcinoma of the thyroid. J Exp Med 1976; 143:993-98.

27. Polliak A, Freund U Mixed papillary and follicular carcinoma of the thyroid gland with stromal amyloid. Am J Clin Pathol 1970; 53:592-95.

28. Valenta LJ, Michelbechet $M$; Mattson JC, Singer FR Microfollicular thyroid carcinoma of the thyroid. Cancer 1977; 39:1573-586.

29. Kakudo K, Miyauchi A, Takai S, Katayama S,Kuma K, Kitamura $\mathrm{H} \mathrm{C}$-cell carcinoma of the thyroid-papillary type. Acta Pathol Jpn 1979; 29:653-59.

30. Normann T, Johannessen JV, Gautvik KM, Olsen BR, Brennhovd IO Medullary carcinoma of the thyroid, diagnostic problems. Cancer 1976; 38:366-77.

31. Harach HR. Williams ED Glandular (tubular and follicular) variants of medullary carcinoma of the thyroid. Histopathology 1983; 7:83-97.

32. Bussolati $G$. Monga $G$ Medullary carcinoma of the thyroid with atypical patterns. Cancer 1979; 44:1769-777.

33. Hales $M$, Rosenau W, Okerlund W, Galante M Carcinoma of the thyroid with a mixed medullary and follicular pattern. Cancer 1982; 50: 1352-359.

34. Ljungberg $O$. Ericsson UB, Bondeson L A compound follicular-parafollicular cell carcinoma of the thyroid: a new tumor entity? Cancer 1983; 52: 1053-61.

35. Pfaltz M. Hedinger CE, Muhlethaler JP Mixed medullary and follicular carcinoma of the thyroid. Virchows Arch (Pathol Anat) 1983; 400:5-93.

36. Martineli G, Bazzocchi F, Govoni E, Santini D Anaplastic type of medullary thyroid carcinoma. Virchows Arch (Pathol Anat) 1983; 400:67-7.

37. Mendelsohn G, Bigner SH, Eggleston JC, Baylin SB, Wells SA Jr Anaplastic variants of medullary thyroid carcinoma. A light microscopic and immunohistochemical study. Am J Surg Pathol 1980; 4:333-41.

38. Holm, R; Sobrinho-Simões, M; Nesland, JM; Sambade, C; Johannessen, JV Medullary thyroid carcinoma with thyroglobulin Immunoreactivity. A special entity? Lab Invest $1987 ; 57: 258-68$.

39. Lips KJM, Veer JvdS; Struyvenberg A, Alleman A, Leo JR. Wittebol P. PFGM, Hackeng WHI Bilateral occurrence of pheochromocytoma in patients with the multiple endocrine neoplasia syndrome type 2A (Sipple's syndrome). Am J Med 1981; 70:1051-60.

40. Hill CS, Ibanez ML, Samaan NA. Ahearn MJ. Clark RL Medullary (solid) carcinoma of the thyroid gland: an analysis of the MDAnderson Hospital experience with patients with the tumor, its special features, and its histogenesis. Medicine 1973; 52:141-71.

41. Carney JA, Sizemore GW, Hayles AB 1978 Multiple endocrine neoplasia, type $2 \mathrm{~b}$ In: loachim HL, ed Patho- biol Annu 8: 105-53.

42. Sizemore GW, Carney JA, Heath III H Epidemiology of medullary carcinoma of the thyroid gland: a 5-year experience (1971-1976). Surg Clin North Am 1977; $57: 633-45$.

43. Melvin, KEW, Tashijian, AH, Miller, HH Studies in familial (medullary) thyroid carcinoma. Rec Prog Horm Res 1972; 28:399-470.

44. Block, MB, Roberts, JP, Kadair, RG, Seyfer, AE; Hull, SF; Nofeldt, FD Multiple endocrine adenomatosis type $\mathrm{llb}$ Diagnosis and management. JAMA 1975; 234:710-14.

45. Carney JA, Roth SI, Heath III. H. Sizemore GW. Hayles AB The parathyroid glands in multiple endocrine neoplasia type 2b. Am J Pathol 1980; 99:387-98.

46. Pommier R, Brennan M Medullary Thyroid Carcinoma. The Endocrinologist 1992; 2(6):393-405.

47. Williams ED Medullary Carcinoma the thyroid In: DeGroot, ed. Endocrinology. Philadelphia, W B Saunders $1995 ; 855-70$.

48. Toledo SPA, Abelin NMA, Ezabella MCL, Hayashida CY, Dahia PLM Neoplasias endócrinas múltiplas. In: Wajchenberg, ed. Tratado de Endocrinologia Clínica. 1992; São Paulo, Roca 941-64.

49. Steiner AL, Goodman AD. Powers SR Study of a kindred with pheochromocytoma, medullary thyroid carcinoma, hiperparathyroidism and Cushing's disease: Multiple Endocrine Neoplasia, type 2. Medicine 1968; 47:371409.

50. Brown JS \& Steiner AL Medullary thyroid carcinoma and the syndromes of multiple endocrine adenomas. Disease of Month 1982; (Chic) 28: 1-37.

51. Nunziata $V$, Giovanni $G$, Lettera AM, DÁrm'ento MD, Mancini $M$ Cutaneous lichen amyloidosis associated with endocrine neoplasia type 2A. Henry Ford Hosp Med J 1989; 37:144-46.

52. Gagel RF, Levy ML, Donavan DT, Alford BR, Wheeler T, Tschen JA Multiple endocrine neoplasia type 2a associated with cutaneous lichen Amyloidosis. Ann Intern Med $1989 ; 111: 802-6$.

53. Robinson MF, Furst EJ, Nunziata $V$, et al Characterization of the clinical features of five families with hereditary primary cutaneous lichen amyloidosis and multiple endocrine neoplasia type 2. Henry Ford Hosp Med J 1992; 40:249-52.

54. Ansari NA, Siegel ME, De Quattro V, Gazarian LH Imaging of Medullary Thyroid Carcinoma and Hiperfunctioning Adrenal Medulla using lodine-131 Metaiodobenzylguanidine. J Nucl Med 1986; 27:1858-60.

55. Clarke S, Lazarus $C$. Maisey $M$ Experience in imaging medullary thyroid carcinoma using $99 \mathrm{M}_{\mathrm{T}}(\mathrm{V})$ dimercaptosuccinic acid (DMSA). Henry Ford Hosp Med J 1989; $37: 167-68$.

56. Talpos GB, Jackson CE, Froelich JW, Kambouris AA, Block MA, Tashjian Jr AH Localization of residual medullary thyroid cancer by thallium scintigraphy. Surgery 1985; 98:1189-196.

57. Vuillez JP, Peftier P, Caravel JP, Chezanneau A, Saccavini JC \& Chazal JR Immunoscintigraphy using 111 -In labeled $F$ ( $a b^{\prime}$ ) 2 fragments of anticarcinoembryonic 
antigen monoclonal antibody for detecting recurrences of medullary thyroid carcinoma. J Clin Endocrinol Metab 1992; 74: 157-63.

58. Kwekkeboom DJ, Reubi JC, Lamberts SWJ, Bruining HA Mulder AH, Oei HY, Krenning EP In vivo somatostatin recptor imaging in medullary thyroid carcinoma. J Clin Endocrinol Metab 1993; 76:1413-417.

59. Jackson CE, Tashijan JrAH, Block MA Detection of medullary thyroid cancer by calcitonin assay in families. Ann Intern Med 1973; 78:845-52.

60. Jackson CE, Tashjian JrAH, Block MA Detection of medullary thyroid cancer. Am J Hum Genet 1979; 31:704-10

61. Ezabella MCL, Hayashida CY, Bisi H, Abelin NM, Toledo SPA 1990 Calcitonina como marcador tumoral do carcinoma medular de tiróide. Arq Brasil Endocrinol Metab 34:6-9.

62. Ishikawa N \& Hamada S Association of medullary carcinoma of the thyroid with carcino-embryonic antigen. $\mathrm{Br}$ J Cancer 1976; 34:111-15.

63. Delellis RA, Rule AH, Spiler I, Nathanson L, Tashijan JrAH Wolfe HJ Calcitonin and carcinoembryonic antigen as tumour markers in medullary thyroid carcinoma. Am J Clin Pathol 1978; 70:587-94.

64. Saad MF, Fritsche Jr HA, Samaan NA Diagnostic and prognostic values of carcinoembryonic antigen in medullary carcinoma of the thyroid. J Clin Endocrinol Metab 1984; 58:889-94.

65. Bravo EL, Gifford JrRW Pheochromocytoma: diagnosis, localization and management. N Engl J Med 1984; 311:1298-303.

66. DeLellis RA, Nunnemacher G, Wolfe HJ C-cell hyperplasia. An ultrastructural analysis. Lab Invest 1971; 36:237-48

67. Hayashida CY, Alves VAF, Kanamura CT, Ezabella MCL, Abelin NM, Nicolau W, et al Toledo SPA Immunohistochemistry of medullary thyroid carcinoma and C-cell hyperplasia by an affinity-purified anti-human calcitonin antiserum. Cancer 1993, 72:1356-363.

68. Toledo SPA Carcinoma medular de tireóide. Rev Hosp Clín Fac Med S Paulo 1985; 40:203-4.

69. Gagel RF, Melvin KEW, Tashiian JrAH Natural history of the familial medullary carcinoma-pheochromocytoma syndrome and the identification of preneoplastic stages by screening studies: A five-year report. Trans Assoc Am Phys 1975; 88:177-91.

70. Graze K, Spiler IJ, Tashjian JrAH, Melvin KEW, Cervi Skinner S, Gagel RF, Natural history of familial medullary thyroid carcinoma. Effect of a program for early diagnosis. N Engl J Med 1978; 299:980-85

71. Ponder BAJ Screening for familial medullary thyroid carcinoma: a review. J R Soc Med 1984; 77:585-94.

72. Ezabella MCL Carcinoma medular de tiróide: testes dinâmicos da secreção de calcitonina em familiares de afetados. Dissertação de mestrado FMUSP 1988.

73. Sizemore GW \& Go VLM Stimulation tests for diagnosis of medullary thyroid carcinoma. Mayo Clin Proc 1975 50:53-6.

74. Tellenius-Berg $M$. Almqvist S, Berg B, Hedner P, Inge- mansson S, Tibblin S, Wasthed B Screening for medullary carcinoma of the thyroid in families with Sipple's syndrome: evaluation of new stimulation tests. Eur J Clin Invest 1977; 7:7-16.

75. Cooper CW, Schwesinger WH, Ontjes DA Thyrocalcitonin: stimulation of secretion by pentagastrin. Science $1971 ; 172: 1238-40$

76. Rude RK \& Singer FR Comparison of serum calcitonin levels after a 1-minute calcium injection and after pentagastrin injection in the diagnosis of medullary thyroid carcinoma. J Clin Endocrinol Metab 1977; 44:980-983.

77. Gagel RF, Tashijan JrAH, Cummings T, Papathanasopoulos N. Kaplan MM. Delellis RA, et al Reichlin S The clinical outcome of prospective screening for multiple endocrine neoplasia type $2 \mathrm{~A}$ An 18-year experience. N Engl J Med 1988; 318:478-84.

78. Graham SM, Genel M, Touloukian RJ, Barwick KW, Gertner JM. Torony $C$ Provocative testing for occult medullary carcinoma of the thyroid: findings in seven children with multiple endocrine neoplasia type lla. J Pediat Surg 1987; 22:501-503.

79. Abelin NM, Dahia P, Martin R, Kato S, Toledo SPA Diagnosis of medullary carcinoma of the thyroid (CMT) using calcitonin (CT) polyclonal radioimmunoassay: criteria for the normal values and pathological levels. Arq Bras Endoc Melab 1994; 38:75.

80. Borges MF, Toledo SPA, Abelin NM, Cunha DF, Barros MAE Calcitonin (CT) deficiency in chronic Hashimoto's thyroiditis (CHT). Illrd European Congress of Endocrinology 1994; $130(2): 140$.

81. Ezabella MCL, Hayashida CY, Bisi H, Leite MOR, Borelli A Abelin NM, et al Toledo SPA Detecção precoce do carcinoma medular de tireóide na neoplasia endócrina múltipla tipo II. Rev Hosp Clín Fac Med S Paulo 1990; 45: 105-109.

82. Ezabella MCL, Hayashida $\mathrm{CY}$, Bisi $\mathrm{H}$, Abelin NM, Brandão LG. Toledo SPA Detecção precoce do carcinoma medular de tireóide. Arq Bras Endocrinol Metab 1990; 34:34-6.

83. Mendonça BB, Bloise W, Bricks F, Bisi H, Nicolau W. Alves VAF, Lima S Cushing's Syndrome due to ectopic ACTH secretion by bilateral pheochromocytomas in multiple endocrine neoplasia type 2A, N Engl J Med 1988; 319:1610-11

84. Bisi $H$, Fernandes VSO, Camargo RYA, Koch L, Abdo AH Brito T The prevalence of unsuspected thyroid pathology in 300 sequencial autopsies, with special reference to the incidental carcinoma. Cancer 1989; 64:1888-93.

85. Chi DD, Toshima K, Donis-Keller $H$, Wells SA, Jr Predictive testing for multiple endocrine neoplasia type 2A (MEN $2 A$ ) based on the detection of mutations in the RET protooncogene. Surgery 1994; 116:124-133.

86. Wells SA, Jr, Chi DD, Toshima K, Dehner LP, Coffin CM, Dowton SB, Predictive DNA testing and prophylactic thyroidectomy in patients at risk for multiple endocrine neoplasia type 2A. Ann Surg 1994; 220:237-250.

87. Calmettes C. Ponder BAJ, Fischer JA, Raue F Early diagnosis of the multiple endocrine neoplasia type 2 syndrome: consensus statement. Eur J Clin Invest 1992; 22:755-60. 
88. Wells SA, Jr New approaches to the patient with medullary carcinoma of the thyroid gland. Thyroid Today 1994; 17:1-9.

89. Brunt LM, Wells SAJr Advances in the diagnosis and treatment of medullary thyroid carcinoma. Surg Clin North Amer 1987; 67:263-79.

90. Kallinowski $F$, Buhr $H J$, Meybier $H$, Eberhandt $M$, Herfarth C Medullary carcinoma of the thyroid - therapeutic strategy derived from fifteen years of experience. Surgery 1993; 114(3):491-96.

91. Samaan NA, Yang KPP, Schultz P, Hickey RC Diagnosis, management and pathogenetic studies in medullary thyroid carcinoma syndrome. Henry Ford Hosp Med J 1989; 37: 132-37

92. Snow KJ, Boyd Alll Management of individual tumor syndromes: medullary thyroid carcinoma and hyperparathyroidism. Endocrinol Metab Clin North Am 1994; 23:157-66.

93. Block MA, Jackson CE, Tashijian JrAH, Management of parathyroid glands in surgery for medullary thyroid carcinoma. Arch Surg 1975; 110:617-24.

94. Mallette LE Management of hyperparathyroidism in the multiple endocrine neoplasia syndromes and other familial endocrinopathies. Endocrinol Metab Clin North Am 1994; 23: 19-36.

95. Steinfeld AD The role of radiation therapy in medullary carcinoma of the thyroid. Radiology $1977 ; 123: 745-6$.

96. Samaan NA, Schultz PN, Hickey RC Medullary thyroid carcinoma: Prognosis of familial versus sporadic disease and the role of radiotherapy. J Clin Endocrinol Metab 1988; 67:801-5.

97. Gottlieb JA, Hill CSJr Chemotherapy of thyroid cancer with adriamycin. New Eng J Med 1974; 290: 193-7.

98. Wiu LT, Averbuch SD, Ball DW, Bustros A, Baylin SB, McGuire WP Treatment of advanced medullary thyroid carcinoma with a combination of cyclophosphamide, vincristine and dacarbazine. Cancer 1994; 73:432-6.

99. Geelhoed SW, Bass LB, Mertz SL, Becker KL Somatostatin analog: Effects on hipergastinemia and hypercalcitoninemia. Surgery 1986; 100(6):962-70.

100. Libroia A, Verga U, Sacco GD, Piolini M. Muratori F Use of somatostatin analog SMS-201-995 in medullary thyroid carcinoma. Henry Ford Hosp Med J 1989; 37: 15 1-3.

101. McEwan LA, Shapiro B. Sisson JC, Beierwaltes H, Ackery DM Radioiodobenzylguanidine for the scintigraphic location and therapy of adrenergic tumours. Semin Nucl Med 1985; 15:134-53.

102. Troncone L, Rufini V. De Rosa $G$, Testa A Diagnostic and therapeutic potential of new radiopharmaceutical agents in medullary thyroid carcinoma. Henry Ford Hosp Med J 1989; 37:178-84.

103. Martin RCT, Ezabella MCL, Toledo SPA Niveis de calci tonina em cultura de tecido de carcinoma medular de tireóide. Rev Hosp Clin Fac Med S Paulo 1985; 40:220-22.

104. Martin RCT, Toleḍo SPA Dinâmica da secreção de calcitonina em cultura de tecido de carcinoma medular de tireóide. Rev Hosp Clin Fac Med S Paulo 1989; 44:36-39.
105. Jackson CE, Talpos GB, Kambouris A, Yozz JB, Tashijan $A H$. Block MA The clinical course after definitive operation for medullary thyroid carcinoma. Surgery 1983; 94:995-1001.

106. Van Heerden JA, Grant CS, Gharib H, Hay ID, Ilstrup DM Long-term course of patients with persistent hypercalcitoninemia after apparent curative primary surgery for medullary thyroid carcinoma. Ann Surg 1990; 212:395-401.

107. Sandrock D, Blossey HC, Steinroeder M, Munz DL Contribution of different scintigraphic techniques to the management of medullary thyroid carcinoma. Henry Ford Hosp Med J 1989; 37: 173-74.

108. Cabezas RC, Berna L, Estarcj M, Carrio I. Ameijeira AG Localization of metastases from medullary thyroid carcinoma using differents methods. Henry Ford Hospital Med 1989; 37: 169-72

109. Clarke S, Lazarus C. Maisey M 1989 Experience in imaging medullary thyroid carcinoma using $99 \mathrm{mTc}(V)$ dimercaptosuccinic acid (DMSA). Henry Ford Hosp Med J 37:167-68.

110. Ben MRAD, Grardet P, Roche A, Rougien P, Calmettes C. Motte P. Parmentier C Values of venous catheterization and calcitonin studies in the treatment and management of clinically inapparent medullary thryroid carcinoma. Cancer 1989; 63:133-38.

111. Gautvik KM, Talle K, Hager B, Jorgensen OG \& Magne AAS Early liver metastases in patients with medullary carcinoma of the thyroid gland. Cancer 1989; 63:17580

112. Raue KF, Raue F, Buhr HJ, Baldauf $G$, Lorenz D, Ziegler $R$ Localization of occult persisting medullany thyroid carcinoma before microsurgical reoperation: high sensitivity of selective venous catheterization. Thyroid 1992; 2:113-17.

113. Block MA, Jackson CE, Tashjian AH Management of occult medullary thyroid carcinoma. Arch Surg 1986; 1 13:368-72

114. Tisell LE, Hansson $G$, Jansson S, Salader H Reoperation in the treatment of asymptomatic metastasizing medullary thyroid carcinoma. Sugery 1986; 99:60-6.

115. Tisell LE, Hansson $G$, Jansson $S$ Surgical treatment of medullary carcinoma of the thyroid. Horm Metab Res 1989; (Suppl) 21:29-31.

116. Buhr HJ, Lehnert T, Raue F New operative strategy in the treatment of metastasizing medullary carcinoma of the thyroid. Europ J Surg Oncol 1990; 16:366-69.

117. Buhr HJ, Kallinowski F, Raue F, Raue KF, Herarth $C$ Microsurgical neck dissection for occult metastasizing medullary thyroid carcinoma. Cancer 1993; 72:368593.

118. Moley JF, Wells SA, Dilley WG Tisel LE Reoperation for recurrent or persistent medullary thyroid cancer. Surgery 1993; 114(6): 1090-96.

119. Saad MF, Ordonez NG, Rashid RK, Quido JJ, Hill Jr CS, Hickey RC, Samaan NA Medullary carcinoma of the thyroid A study of the clinical features and prognostic factors in 161 patients. Medicine 1984; 63:319-42.

120. Bergholm $V$. Adami $H O$, Bergstrom R, Bäckdahl $M$, Akerstrom $G$ The Swedish MTC study group - Long term survival in sporadic and familial medullary thy- 
roid carcinoma with special reference to clinical characteristics as prognostic factor. Acta Chir Scand 1990; 156:37-46.

121. Ellenhorn JDI, Shah JP, Brennan F Impact of therapeutic regional lymph node dissection for medullary carcinoma of the thyroid gland. Surgery 1993; 114:1078-82.

122. Pacini F, Elisei R, Anelli S, Basolo F, Cola A, Pinchera A Somatostatin in medullary thyroid cancer. Cancer 1989; 63: $1189-1195$.

\section{Endereço para correspondência:}

Disciplina de Endocrinologia

Hospital das Clínicas da Faculdade

de Medicina da USP

Caixa Postal 3.671

01060-970 São Paulo-SP 